\title{
Pathway-based detection of idiopathic pulmonary fibrosis at an early stage
}

\author{
GUOJUN ZHOU ${ }^{1}$, FANGXIA ZHANG ${ }^{2}$, YUFANG LIU ${ }^{3}$ and BIN SUN ${ }^{1}$ \\ Departments of ${ }^{1}$ Emergency; ${ }^{2}$ Cardiology and ${ }^{3}$ Gynaecology and Obstetrics, \\ Medical University Hospital of Binzhou, Binzhou, Shandong 256600, P.R. China
}

Received January 8, 2016; Accepted January 6, 2017

DOI: $10.3892 / \mathrm{mmr} .2017 .6274$

\begin{abstract}
Idiopathic pulmonary fibrosis (IPF) is the most common interstitial pneumonia and the most aggressive interstitial lung disease. Usually, IPF is confirmed by the histopathological pattern of typical interstitial pneumonia and requires an integrated multidisciplinary approach from pulmonologists, radiologists and pathologists. However, these diagnoses are performed at an advanced stage of IPF. At present, pathway-based detection requires investigation, as it can be performed at an early stage of the disease. The aim of the present study was to find an effective method of diagnosing IPF at an early stage. Microarray data forE-GEOD-33566 were downloaded from the ArrayExpress database. Human pathways were downloaded from Kyoto Encyclopedia of Genes and Genomes (KEGG) pathway database. An individual pathway-based method to diagnose IPF at an early stage was introduced. Pathway statistics were analyzed with an individualized pathway aberrance score. P-values were obtained with different methods, including the Wilcoxon test, linear models for microarray data (Limma) test and attract methods, generating three pathway groups. Support vector machines (SVM) were used to identify the best group for diagnosing IPF at an early stage. There were 106 differential pathways in Wilcoxon-based KEGG Pathway ( $>5$ ) group, 100 in the Limma-based KEGG Pathway $(n>5)$ group, and seven in the attract-based KEGG Pathway $(n>5)$ group. The pathway statistics of these differential pathways in three groups were analyzed with linear SVM. The results demonstrated that the Wilcoxon-based KEGG Pathway $(n>5)$ group performed best in diagnosing IPF.
\end{abstract}

\section{Introduction}

Idiopathic pulmonary fibrosis (IPF) is the most common of the interstitial pneumonias and the most aggressive interstitial

Correspondence to: Mr. Bin Sun, Department of Emergency, Medical University Hospital of Binzhou, 661 Huanghe Road, Bincheng, Binzhou, Shandong 256600, P.R. China

E-mail: sunbinmed@yeah.net

Key words: idiopathic pulmonary fibrosis, diagnose, test, differential pathway, support vector machines lung disease (1). The etiology of IPF still remains to be elucidated and thus, a successful treatment remains to be identified. The disease is more common in males, particularly those aged between 50 and 70 (2), and the incidence of IPF rises markedly with age. The prevalence of IPF ranges between 13 cases per 100,000 for women to 20 cases per 100,000 for men and the figures are increasing (3). The onset of clinical symptoms is insidious, including shortness of breath on exertion and a dry cough, and certain patients experience an initial flu-like malaise (1), leading to a late diagnosis if ignored.

Usually IPF is confirmed by the histopathological pattern of usual interstitial pneumonia, and requires an integrated multidisciplinary approach from pulmonologists, radiologists and pathologists. The common measurements include high-resolution computed tomography, surgical lung biopsy and radiologic diagnosis. However, these diagnoses are performed at a late stage of IPF and are not useful in proposing a plan of treatment.

A recent genetic study (4) assessed early-stage pulmonary fibrosis as the majority of these mutations are present at birth, predating disease development, and thus can provide insights into the early stages. A study of genetic associations (5) holds promise in exhibiting the connections between early-stage and advanced disease. Although progress has been made in the field of IPF genetics in identifying common variants that are associated with IPF diagnosis, rare variants remain to be analyzed. The use of genetics in early IPF detection remains in its infancy.

It has been demonstrated (6) that numerous critical genes and pathways are deregulated during the initiation and progression of a cancer, certain studies $(7,8)$ have identified differential expressed genes in IPF and several studies $(7,9)$ have analyzed pathways in IPF, however they were non-uniform. Identifying pathways that are deregulated in patients with cancer may be useful in identifying cancer from unknown samples. A number of methods have been proposed to identify differential pathways, including the attract method (10), personal pathway deregulation score (11) and individualized pathway aberrance score (12). Personalized identification of differential pathways provides pathway interpretation in a single sample with accumulated normal data.

Support vector machines (SVM) are among the most powerful classification and prediction methods, first developed by Cherkassky (13). They are used in a wide range of scientific applications (14), including cancer tissue classification (15), 
protein domain classification (16) and splice site prediction (17), due to their great accuracy, their ability to deal with high-dimensional and large datasets, and their flexibility in modeling diverse sources of data (18).

From this perspective, a pathway aberrance analysis to identify and determine the extent of IPF using the peripheral blood transcriptome was performed, with the aim of distinguishing normal individuals from patients with IPF and, additionally, to distinguish the extent of the disease when samples were classified by percent predicted diffusion capacity for carbon monoxide of the lung, however not forced vital capacity (19). Three methods were employed to identify differential pathways. To analyze the feasibility of pathway-based diagnosis in IPF, SVM was introduced.

\section{Materials and methods}

\section{Dataset}

Gene expression data. Microarray data of E-GEOD-33566 (19), together with the annotation files, were downloaded from the ArrayExpress database (https://www.ebi.ac.uk/arrayexpress). The data included 93 patients with IPF and 30 healthy controls. Blood was collected in PAXgene RNA tubes. The platform in this study was A-AGIL-28-Agilent Whole Human Genome Microarray 4x44K 014850 G4112F (85 columnsx532 rows) and the platform was designated. The Peripheral Blood Transcriptome Predicts the Presence and Extent of Disease in Idiopathic Pulmonary Fibrosis, by which the gene expression files were generated. According to the gene ID and symbol in the annotation file of the platform, the gene ID in the microarray was changed to its designation.

Pathway data and preprocessing. All the pathways of Homo sapiens were derived from the Kyoto Encyclopedia of Genes and Genomes (KEGG) Pathway Database (http://www. kegg.jp) (20). In total, 300 pathways, including 6,919 genes, were obtained. To simplify pathway data, pathways containing $<5$ genes were excluded. Eventually, 284 pathways were obtained for further analysis. Genes common to pathways and samples were used in subsequent analysis.

Pathway analysis. The aim of the present study was to analyze the altered pathways in an individual with a disease. The process of this analysis is presented as Fig. 1.

Gene level statistics. Gene data in the normal group were normalized using quantile normalization in the preprocessCore package (21), which generated the mean and standard deviation of gene expression levels. Following the amalgamation of genes in tumor samples with all the normal samples, quantile normalization using mean and standard deviation of the gene expression levels was performed, generating gene level statistics. The formula was:

$$
\mathrm{Z}_{i}=\frac{\mathrm{g}_{\mathrm{T} i}-\operatorname{mean}\left(\mathrm{g}_{\mathrm{nRef}}\right)}{\operatorname{stdev}\left(\mathrm{g}_{\mathrm{nRef}}\right)}
$$

Where $Z_{i}$ symbolized the standardized expression value of the $i$-th gene, and $\mathrm{n}$ represented the number of genes belonging to the pathway. The results obtained were gene level statistics.

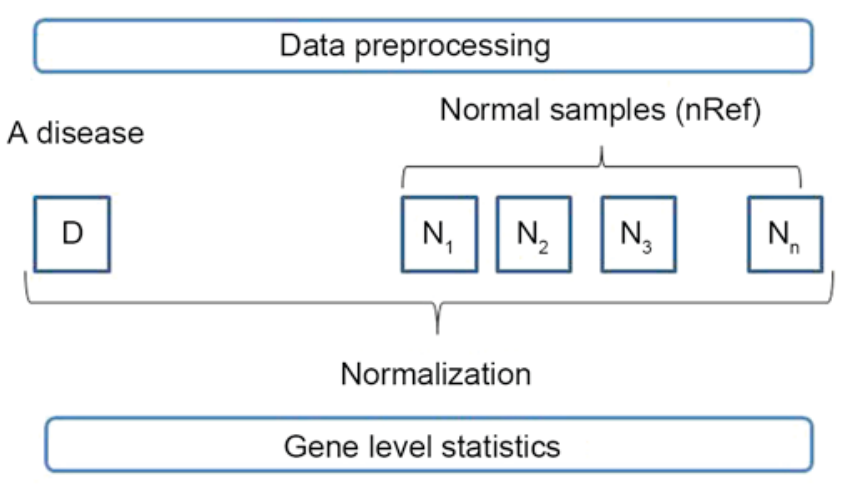

Average $Z: \quad Z_{i}=\frac{g_{T i}-\text { mean }\left(g_{\text {nRef }}\right)}{\operatorname{stdev}\left(g_{\text {nRef }}\right)}$

Pathway statistics

Individual pathway aberrance score $=\frac{\sum_{\mathrm{i}}^{\mathrm{n}} z_{i}}{\mathrm{n}}$

Figure 1. A schematic diagram of the method of individualized pathway analysis.

Pathway level statistics. The statistics for each pathway were calculated by averaging the gene level statistics of all genes belonging to the pathway, thus:

$$
\text { Pathway statistics }=\frac{\sum_{i}^{\mathrm{n}} \mathrm{z}_{i}}{\mathrm{n}}
$$

Where $\mathrm{n}$ represented the number of genes in the pathway and $\mathrm{Z}_{i}$ symbolized the standardized expression value of $i$-th gene in the pathway.

Differential pathway screening. A significance test was performed to assess differential pathways associated with IPF. To identify the best test protocol to assess differential pathways, three pathway groups were constructed for comparison.

Wilcoxon-based KEGG Pathway $(n>5)$ group: The pathway statistics, obtained from the pathways of disease group and normal group, were tested by Wilcoxon (22) with the function:

$$
\begin{aligned}
E(T) & =\frac{n(n+1)}{4} \\
D(T) & =\frac{n(n+1)(2 n+1)}{24} \\
Z & =\frac{T-E(T)}{\sqrt{D(T)}}
\end{aligned}
$$

Where $\mathrm{n}$ is the number of samples.

The significance of the level was corrected by false discovery rate (FDR) (23).

Subsequently, each pathway was allotted a P-value. Those pathways with $\mathrm{P}<0.01$ were considered differential pathways. In total, 106 differential pathways were obtained.

Limma-based KEGG Pathway ( $>5$ ) group: The pathway statistics were performed with Limmae Bayes (24) and top Table functions, generating P-values. In total, 100 differential pathways were screened out with $\mathrm{P}<0.01$.

Attract-based KEGG Pathway $(n>5)$ group: Genes in differential pathways of the Wilcoxon-based KEGG pathway group were subsequently analyzed using the attract method. 
The F-statistics for gene I was calculated by:

$$
F^{(i)}=\frac{\text { MSS }_{i}}{\operatorname{RSS}_{i}}
$$

Where $\mathrm{MSS}_{\mathrm{i}}$ denotes the mean treatment sum of squares:

$$
\mathrm{MSS}_{\mathrm{i}}=\frac{1}{\mathrm{~K}-1} \sum_{\mathrm{k}=1}^{\mathrm{K}} \mathrm{r}_{\mathrm{k}}\left[\mathrm{y}_{\cdot \mathrm{k}}^{(\mathrm{i})}-\mathrm{y}_{\cdot}^{(\mathrm{i})}\right]^{2}
$$

And $\mathrm{RSS}_{\mathrm{i}}$ denotes the residual sum of squares:

$$
\mathrm{RSS}_{\mathrm{i}}=\frac{1}{\mathrm{~N}-\mathrm{K}} \sum_{\mathrm{k}=1}^{\mathrm{K}} \sum_{\mathrm{j}=1}^{\mathrm{r}_{\mathrm{j}}}\left[\mathrm{y}_{\mathrm{jk}}^{(\mathrm{i})}-\mathrm{y}_{. .}^{(\mathrm{i})}\right]^{2}
$$

For pathway $\mathrm{P}$ consisting of $\mathrm{g}_{\mathrm{p}}$ genes, the T-statistic takes the following form:

$$
\mathrm{T}_{\mathrm{p}}=\frac{\left[\frac{1}{\mathrm{~g}_{\mathrm{p}}} \sum_{\mathrm{i}=1}^{\mathrm{g}_{\mathrm{p}}} \mathrm{F}^{(\mathrm{i})}\right]-\left[\frac{1}{\mathrm{G}} \sum_{\mathrm{j}=1}^{\mathrm{G}} \mathrm{F}^{(\mathrm{i})}\right]}{\sqrt{\left(\frac{\mathrm{S}_{\mathrm{p}}{ }^{2}}{\mathrm{~g}_{\mathrm{p}}}\right)+\left(\frac{\mathrm{S}_{\mathrm{G}}{ }^{2}}{\mathrm{G}}\right)}}
$$

Where $\mathrm{G}$ denotes the total number of genes in a pathway and $\mathrm{S}_{\mathrm{P}}{ }^{2}$ and $\mathrm{S}_{\mathrm{G}}{ }^{2}$ were defined as sample aberrances.

Following the performance of the t-test and adjusted with the FDR of Benjamini-Hochberg (25), the pathway statistical value was transformed into P-values. In total, seven pathways with $\mathrm{P}<0.05$ were identified.

SVM analysis. An SVM method was applied to test the analysis results of the three pathway groups and 5-fold cross validation was selected to analyze the SVM model. The pathways statistics of the normal and disease group were amalgamated and divided into two sets, the training and the test set, with a ratio of 6:4. These data were treated with linear SVM, employing the formula:

$$
\mathrm{K}\left(\mathrm{x}, \mathrm{x}_{\mathrm{i}}\right)=\left[\left(\mathrm{x}^{*} \mathrm{x}_{\mathrm{i}}\right)+1\right]^{\mathrm{q}}
$$

Subsequent to classification, the parameters of the area under the receiver operator characteristic (ROC) curve (AUC), accuracy, the Matthews coefficient correlation classification measure (MCC), the degree of true negative identification specificity (specificity) and the degree of true positive identification sensitivity (sensitivity) were ascertained.

\section{Results}

Differential pathways. The original KEGG pathway database contains 300 pathways and 6,919 genes. Pathways with $<5$ genes were deleted, generating a KEGG Pathway $(n>5)$ group containing 284 pathways and 4,303 genes. In comparing the healthy $(n=30)$ and diseased $(n=93)$ lung samples, differential pathways were identified using three methods.

In the Wilcoxon-based KEGG Pathway ( $>55)$ group, 106 differential pathways were identified, the largest number of the three groups. By ranking pathways with P-values, five pathways with the least $\mathrm{P}$-values and gene number are presented in Table I. The P-value can be regarded as an indicator of the extent of the disease. The first differential pathway with the least P-value was 'Amoebiasis', indicating that it was among
Table I. The top five ranked differential pathways with the least $\mathrm{P}$-values in the Wilcoxon-based KEGG pathway group $(n>5)$.

\begin{tabular}{lcc}
\hline Differential pathway & P-value & Geneno. \\
\hline Amoebiasis & 0.000151 & 60 \\
Bladder cancer & 0.000186 & 29 \\
Type II diabetes mellitus & 0.000236 & 30 \\
Primary immunodeficiency & 0.000386 & 31 \\
Histidine metabolism & 0.000386 & 9 \\
\hline
\end{tabular}

KEGG, Kyoto Encyclopedia of Genes and Genomes; Geneno., the number of genes in the pathway.

Table II. The top five ranked differential pathways with P-values in the Limma-based KEGG Pathway group $(n>5)$.

\begin{tabular}{llc}
\hline Differential pathway & P-value & Genesno. \\
\hline Amoebiasis & 0.0000684 & 60 \\
Bladder cancer & 0.0000684 & 9 \\
Type II diabetes mellitus & 0.00022 & 31 \\
Primary immunodeficiency & 0.000405 & 30 \\
Histidine metabolism & 0.000405 & 38
\end{tabular}

Limma, linear models for microarray data; KEGG, Kyoto Encyclopedia of Genes and Genomes; Genesno., the number of genes in the pathway.

Table III. All the differential pathways with P-values in the attract-based KEGG Pathway group ( $>5$ ).

\begin{tabular}{lcc}
\hline Differential pathways & P-value & Geneno. \\
\hline Ribosome & 0.000072 & 128 \\
Legionellosis & 0.000072 & 48 \\
Pyrimidine metabolism & 0.001157 & 79 \\
Renin-angiotensin system & 0.001157 & 7 \\
B cell receptor signaling & 0.002139 & 70 \\
Oxidative phosphorylation & 0.006775 & 115 \\
Osteoclast differentiation & 0.006775 & 109 \\
\hline
\end{tabular}

KEGG, Kyoto Encyclopedia of Genes and Genomes; Geneno., the number of genes in the pathway.

the pathways most susceptible to disease. It is caused by an extracellular protozoan parasite that invades the intestinal epithelium and belongs to infectious diseases. The pathway of 'bladder cancer' demonstrates that the disease causes urinary system lesion. The other three pathways are involved in basic metabolism in the body.

The Limma-based KEGG Pathway ( $\mathrm{n}>5$ ) group contained 100 differential pathways, six fewer than the Wilcoxon-based KEGG Pathway $(n>5)$ group. The top five ranked pathways with the least P-values and gene number are presented in 
Table IV. Comparison of the test sets of the three differential pathway groups classified by the method ofsupport vector machines.

\begin{tabular}{lccc}
\hline Parameter & $\begin{array}{c}\text { Limma-based } \\
\text { KEGG pathway }\end{array}$ & $\begin{array}{c}\text { Wilcoxon-based } \\
\text { KEGG pathway }\end{array}$ & $\begin{array}{c}\text { Attract-based } \\
\text { KEGG pathway }\end{array}$ \\
\hline Negative samples & 14 & 14 & 14 \\
Positive samples & 36 & 36 & 36 \\
TN & 7 & 8 & 0 \\
FP & 7 & 6 & 36 \\
TP & 31 & 33 & 0 \\
FN & 5 & 0.74 & 0.50 \\
AUC & 0.68 & 82.00 & 72.00 \\
Accuracy & 76.00 & 0.53 & 0.00 \\
MCC & 0.38 & 0.57 & 0.00 \\
Specificity & 0.50 & 0.92 & 1.00 \\
Sensitivity & 0.86 & 36 & \\
\hline
\end{tabular}

Limma, linear models for microarray data; KEGG, Kyoto Encyclopedia of Genes and Genomes; TN, true negative; FP, false positive; TP, true positive; FN, false negative; AUC, the area under the ROC curve; ROC, receiver operator characteristic; MCC, the Matthews coefficient correlation classification measure.

Table II. Notably, four pathways were the same as in the Wilcoxon-based KEGG Pathway ( $\mathrm{n}>5$ ) group. The exception is 'Notch signaling pathway', an intercellular signaling mechanism essential for correct embryonic development.

The attract-based KEGG Pathway $(\mathrm{n}>5$ ) group contained seven differential pathways, and was the smallest group. These differential pathways were the same as seven of the differential pathways in Wilcoxon-based KEGG Pathway $(n>5)$ group, but none of them were in the top five pathways of the latter group in P-values. The pathways with P-values and gene number are presented in Table III. The seven pathways represented the core pathways that reflected the disease and may aid analysis of the disease. The first ranked pathway was 'Ribosome', which is responsible for genetic information processing and translation. The 'Legionellosis' pathway is associated with a potentially fatal infectious disease. 'Pyrimidine metabolism' is responsible for nucleotide metabolism. The 'Renin-angiotensin system' pathway is a peptidergic system with endocrine characteristics concerned with the regulation of blood pressure and hydroelectrolytic balance. The 'B cell receptor signaling' pathway is involved in the immune system. The 'Oxidative phosphorylation' pathway is part of energy metabolism.

SVM analysis. To obtain the best performing pathway group, linear SVM analysis was adopted. In each differential pathway group, pathways in the normal and disease groups were divided into two sets, the training and the test set, with a ratio of $6: 4$. Several parameters were analyzed to compare the four pathway groups, including AUC, accuracy, specificity, sensitivity, MCC, true negative, false positive, true positive and false negative. The test set of the differential pathway groups with parameters is presented in Table IV.

According to the SVM results, the Wilcoxon-based KEGG Pathway $(n>5)$ group performed the best, with all the parameters better than the other two groups.

\section{Discussion}

A method to diagnose IPF at an early stage is required. Since the field of IPF genetics has made significant progress in identifying common variants that are confidently associated with IPF diagnosis, a gene-based pathway aberrance analysis may aid the detection of IPF at an early stage.

In the present study, three pathway groups were constructed; a Wilcoxon-based KEGG Pathway $(n>5)$ group, a Limma-based KEGG Pathway $(n>5)$ group and an attract-based KEGG Pathway $(n>5)$ group. Different groups were obtained due to the different test methods deployed in pathway statistics and the quantity of differential pathways in the three groups also differed; the Wilcoxon-based KEGG Pathway $(n>5)$ group possessed the greatest number of pathways, the Limma-based KEGG Pathway $(n>5)$ group possessed fewer pathways and the attract-based KEGG Pathway $(n>5)$ group the least number of pathways. The attract-based KEGG Pathway $(n>5)$ group contained only seven differential pathways, far fewer than the other two groups. Differential pathways reflected the core metabolisms that were most influenced by the disease, however the large number of differential pathways identified suggested further evaluation and study is required in order to fully elucidate the mechanism.

The SVM method (26), which has been demonstrated to possess a high identification rate in numerous datasets, was introduced to perform the comparison. According to the SVM results, the Wilcoxon-based KEGG Pathway ( $>5$ ) group performed the best, with all parameters better than the other two groups.

To identify which group performed best in diagnosing IPF with differential pathways, a classifier SVM was introduced. The results demonstrated that the Wilcoxon-based KEGG Pathway $(n>5)$ group performed the best, with the parameters of AUC, accuracy, MCC, specificity and sensitivity. It is therefore suggested that this pathway group reflected the occurrence of IPF more exactly. The top five pathways that were most prone 
to alter in IPF were 'Amoebiasis', 'Bladder cancer', 'Type II diabetes mellitus', 'Primary immunodeficiency' and 'Histidine metabolism'.

The 'Amoebiasis'pathway is involved in a type of infectious disease. The pathogenesis of amoebiasis begins with parasite attachment and disruption of the intestinal mucus layer, followed by apoptosis of host epithelial cells. The parasite can cause extra intestinal infection, including amoebic liver abscesses, by evading the immune response (27). That the 'Amoebiasis' pathway was inhibited in IPF was identified by Nance et al (28). In the present study, the 'Amoebiasis' pathway in the disease group was demonstrated to be significantly different from the normal group, which was consistent with the result of Nance et al (28).

The 'Bladder cancer' pathway is responsible for bladder cancer. This pathway was significantly altered in IPF, which may be the result of the deregulation of a regulator, caveolin-1, since caveolin-1deregulation has been associated with several human diseases (29-32). It has been demonstrated that caveolin-1 mRNA expression is low in IPF (33), however is high in bladder cancer (32).

The 'Type II diabetes mellitus' pathway was identified altered in IPF. Among various lifestyle-associated diseases, diabetes mellitus is a frequent complication in patients with IPF and may increase the risk of IPF (34).

'Primary immunodeficienies' are a heterogeneous group of disorders, which affect cellular and humoral immunity or non-specific host defense mechanisms mediated by complement proteins and cells (35). It has been previously demonstrated (36) that in a severe combined immunodeficiency bleomyc in mouse model of fibrosis, human fibrocytes are also trafficked to the lung, the primary area of injury.

In summary, differential pathways can be used in diagnosis of IPF at an early stage, and the best method analyzed by SVM is by making use of the significant differential pathways identified in the Wilcoxon-based KEGG Pathway $(n>5)$ group.

\section{Acknowledgements}

The authors would like to thank Honghui Biotechnology Co., Ltd. (Shandong, China) for help in information analysis.

\section{References}

1. Michaelson JE, Aguayo SM and Roman J: Idiopathic pulmonary fibrosis: A practical approach for diagnosis and management. Chest 118: 788-794, 2000.

2. Woodcock HV and Maher TM: The treatment of idiopathic pulmonary fibrosis. F1000prime Rep 6: 16, 2014.

3. Agabiti N, Porretta MA, Bauleo L, Coppola A, Sergiacomi G, Fusco A, Cavalli F, Zappa MC, Vignarola R, Carlone S, et al: Idiopathic pulmonary fibrosis (IPF) incidence and prevalence in Italy. Sarcoidosis Vasc Diffuse Lung Dis 31: 191-197, 2014.

4. Putman RK, Rosas IO and Hunninghake GM: Genetics and early detection in idiopathic pulmonary fibrosis. Am J Respir Crit Care Med 189: 770-778, 2014.

5. Levine DM, Ek WE, Zhang R, Liu X, Onstad L, Sather C, Lao-Sirieix P, Gammon MD, Corley DA, Shaheen NJ, et al: A genome-wide association study identifies new susceptibility loci for esophageal adenocarcinoma and Barrett's esophagus. Nat Genet 45: 1487-1493, 2013.

6. Bild AH, Yao G, Chang JT, Wang Q, Potti A, Chasse D, Joshi MB, Harpole D, Lancaster JM, Berchuck A, et al: Oncogenic pathway signatures in human cancers as a guide to targeted therapies. Nature 439: 353-357, 2006.
7. Nance T, Smith KS, Anaya V, Richardson R, Ho L, Pala M Mostafavi S, Battle A, Feghali-Bostwick C, Rosen G and Montgomery SB: Transcriptome analysis reveals differential splicing events in IPF lung tissue. PLoS One 9: e97550, 2014.

8. Deng N, Sanchez CG, Lasky JA and Zhu D: Detecting splicing variants in idiopathic pulmonary fibrosis from non-differentially expressed genes. PLoS One 8: e68352, 2013.

9. Boon K, Bailey NW, Yang J, Steel MP, Groshong S, Kervitsky D, Brown KK, Schwarz MI and Schwartz DA: Molecular phenotypes distinguish patients with relatively stable from progressive idiopathic pulmonary fibrosis (IPF). PLoS One 4: e5134, 2009.

10. Mar JC, Matigian NA, Quackenbush J and Wells CA: attract: A method for identifying core pathways that define cellular phenotypes. PLoS One 6: e25445, 2011.

11. Drier Y, Sheffer M and Domany E: Pathway-based personalized analysis of cancer. Proc Natl Acad Sci USA 110: 6388-6393, 2013.

12. Ahn T, Lee E, Huh N and Park T: Personalized identification of altered pathways in cancer using accumulated normal tissue data. Bioinformatics 30: i422-i429, 2014.

13. Cherkassky V: The nature of statistical learning theory . IIEEE Trans Neural Netw 8: 1564, 1997.

14. Ben-Hur A, Ong CS, Sonnenburg S, Schölkopf B and Rätsch G: Support vector machines and kernels for computational biology. PLoS Comput Biol 4: e1000173, 2008.

15. Furey TS, Cristianini N, Duffy N, Bednarski DW, Schummer M and Haussler D: Support vector machine classification and validation of cancer tissue samples using microarray expression data. Bioinformatics 16: 906-914, 2000.

16. Karchin R, Karplus $\mathrm{K}$ and Haussler D: Classifying G-protein coupled receptors with support vector machines. Bioinformatics 18: 147-159, 2002.

17. Sonnenburg S, Schweikert G, Philips P, Behr J and Rätsch G: Accurate splice site prediction using support vector machines. BMC Bioinformatics 8 (Suppl 10): S7, 2007.

18. Müller KR, Mika S, Rätsch G, Tsuda K and Schölkopf B: An introduction to kernel-based learning algorithms. IEEE Trans Neural Net 12: 181-201, 2001

19. Yang IV, Luna LG, Cotter J, Talbert J, Leach SM, Kidd R, Turner J, Kummer N, Kervitsky D, Brown KK, et al: The peripheral blood transcriptome identifies the presence and extent of disease in idiopathic pulmonary fibrosis. PLoS One 7: e37708, 2012.

20. Kanehisa M, Goto S, Sato Y, Furumichi M and Tanabe M: KEGG for integration and interpretation of large-scale molecular data sets. Nucleic Acids Res 40 (Database issue): D109-D114, 2012.

21. Bolstad B: preprocessCore: A collection of pre-processing functions. Bioconductor, 2013.

22. Gehan EA: A generalized wilcoxon test for comparing arbitrarily singly-censored samples. Biometrika 52: 203-223, 1965.

23. Benjamini Y, Drai D, Elmer G, Kafkafi N and Golani I: Controlling the false discovery rate in behavior genetics research. Behav Brain Res 125: 279-284, 2001.

24. McCarthy DJ and Smyth GK: Testing significance relative to a fold-change threshold is a TREAT. Bioinformatics 25: 765-771, 2009.

25. Lazar C, Taminau J, Meganck S, Steenhoff D, Coletta A, Molter C, de Schaetzen V, Duque R, Bersini H and Nowé A: A survey on filter techniques for feature selection in gene expression microarray analysis. IEEE/ACM Trans Comput Biol Bioinform 9: 1106-1119, 2012.

26. Papadonikolakis M and Bouganis CS: Novel cascade FPGA accelerator for support vector machines classification. IEEE Trans Neural Netw Learn Syst 23: 1040-1052, 2012.

27. Lejeune M, Rybicka JM and Chadee K: Recent discoveries in the pathogenesis and immune response toward Entamoeba histolytica. Future Microbiol 4: 105-118, 2009.

28. Nance T, Smith KS, Anaya V, Richardson R, Ho L, Pala M, Mostafavi S, Battle A, Feghali-Bostwick C, Rosen G and Montgomery SB: Transcriptome analysis reveals differential splicing events in IPF lung tissue. PLoS One 9: e92111, 2014.

29. Wang XM, Zhang Y, Kim HP, Zhou Z, Feghali-Bostwick CA, Liu F, Ifedigbo E, Xu X, Oury TD, Kaminski N and Choi AM: Caveolin-1: A critical regulator of lung fibrosis in idiopathic pulmonary fibrosis. J Exp Med 203: 2895-2906, 2006.

30. Williams TM and Lisanti MP: Caveolin-1 in oncogenic transformation, cancer, and metastasis. Am J Physiol Cell Physiol 288: C494-C506, 2005. 
31. Sotgia F, Williams TM, Schubert W, Medina F, Minetti C, Pestell RG and Lisanti MP: Caveolin-1 deficiency (-/-) conveys premalignant alterations in mammary epithelia, with abnormal lumen formation, growth factor independence, and cell invasiveness. Am J Pathol 168: 292-309, 2006.

32. Thomas S, Overdevest JB, Nitz MD, Williams PD, Owens CR Sanchez-Carbayo M, Frierson HF, Schwartz MA and Theodorescu D: Src and caveolin-1 reciprocally regulate metastasis via a common downstream signaling pathway in bladder cancer. Cancer Res 71: 832-841, 2011.

33. Nho RS, Peterson M, Hergert $\mathrm{P}$ and Henke CA: FoxO3a (Forkhead Box O3a) deficiency protects idiopathic pulmonary fibrosis (IPF) fibroblasts from type I polymerized collagen matrix-induced apoptosis via caveolin-1 (cav-1) and Fas. PLoS One 8: e61017, 2013
34. Enomoto T, Usuki J, Azuma A, Nakagawa T and Kudoh S: Diabetes mellitus may increase risk for idiopathic pulmonary fibrosis. Chest 123: 2007-2011, 2003.

35. Geha RS, Notarangelo LD, Casanova JL, Chapel H, Conley ME, Fischer A, Hammarström L, Nonoyama S, Ochs HD, Puck JM, et al: Primary immunodeficiency diseases: An update from the International union of immunological societies primary immunodeficiency diseases classification committee. J Allergy Clin Immunol 120: 776-794, 2007.

36. Phillips RJ, Burdick MD, Hong K, Lutz MA, Murray LA, Xue YY, Belperio JA, Keane MP and Strieter RM: Circulating fibrocytes traffic to the lungs in response to CXCL12 and mediate fibrosis. J Clin Invest 114: 438-446, 2004 